\title{
FITOTECNIA
}

\section{PODA APICAL, DENSIDADE DE PLANTAS E COBERTURA PLÁSTICA DO SOLO NA PRODUTIVIDADE DO TOMATEIRO EM CULTIVO PROTEGIDO $\left(^{\mathbf{1}}\right)$}

\author{
JÚLIO CÉSAR BOGIANI $\left({ }^{2}\right)$; CIBELE DA SILVA ANTON $\left({ }^{3}\right)$; ALEXSANDER SELEGUINI $\left({ }^{4}\right)$; \\ MAX JOSÉ DE ARAÚJO FARIA JÚNIOR $\left({ }^{5}\right)$; SHIZUO SENO $\left({ }^{6}\right)$
}

\begin{abstract}
RESUMO
O objetivo deste trabalho foi o de avaliar o efeito de duas combinações entre densidade de plantas e altura de poda apical, associados à utilização de cobertura plástica do solo, sobre o desenvolvimento e a produção de frutos do tomateiro, híbrido Duradoro (EMBRAPA), em ambiente protegido. Foram estabelecidos dois sistemas de cultivo com haste única: SC1 - poda apical após a $5^{\mathrm{a}}$ inflorescência, com 20.000 plantas ha-1 e SC2 - poda apical após a $2^{\mathrm{a}}$ inflorescência com 50.000 plantas ha-1; e três condições de cobertura do solo: solo nu; filme de polietileno branco e filme de polietileno preto, adotando-se o delineamento em blocos ao acaso, em esquema de parcelas subdivididas. O experimento foi desenvolvido de junho a novembro de 2003, na UNESP, Câmpus de Ilha Solteira. A adoção do tratamento SC2 determinou maior altura de plantas aos 45 dias após o transplante, maior número de frutos por área e maior produtividade, além da concentração do período de colheita em sete semanas. As coberturas plásticas do solo não influenciaram as variáveis que compõem a produtividade do tomateiro.
\end{abstract}

Palavras-chave: Lycopersicon esculentum; poda apical, densidade, cultivo protegido.

\section{ABSTRACT \\ TIP PRUNING, PLANT DENSITY AND PLASTIC MULCHING IN TOMATO YIELD IN PROTECTED CULTIVATION}

The aim of this work was to evaluate the effect of two combinations between plant density and tip pruning height, associated to the use of plastic mulching, on plants development and fruit yield of the tomato (Duradoro hybrid) grown under protected environment. Two single stem cultivation systems were compared: SC1 - tip pruning after the fifth inflorescence with 20,000 plants ha-1; SC2 - tip pruning after the second inflorescence with 50,000 plants $\mathrm{ha}^{-1}$, and three different soil cover conditions: bare soil, white polyethylene film, $50 \mathrm{~mm}$-thick, and black polyethylene film, $50 \mathrm{~mm}$-thick. The experimental design used was randomized block, in a split plot array. The work was carried out from June to November of 2003, at the UNESP, Ilha Solteira (São Paulo State, Brazil). The treatment SC2 increased plant height at 45 days after the transplant, and reached higher number and fruit yield, with reduction in cropping period. The soil cover with polyethylene mulching did not affect the yield of this crop.

Key words: Lycopersicon esculentum, tip pruning, plant density, protected environment.

$\left({ }^{1}\right)$ Recebido para publicação em 20 de outubro de 2006 e aceito em 20 de agosto de 2007.

$\left({ }^{2}\right)$ Egresso do Curso de Graduação Agronomia da UNESP-Câmpus de Ilha Solteira. (Ex-bolsista FAPESP de Iniciação Científica)

$\left({ }^{3}\right)$ Egressa do Curso de Pós-Graduação Agronomia (Mestrado) da UNESP, Câmpus de Ilha Solteira. Ex-Bolsista da CAPES.

$\left({ }^{4}\right)$ Doutorando do Curso de Pós-Graduação Agronomia da UNESP, Câmpus de Ilha Solteira. Bolsista CAPES.

$\left({ }^{5}\right)$ Departamento de Fitossanidade, Engenharia Rural e Solos, UNESP, Câmpus de Ilha Solteira, Caixa Postal 31, 15385-000 Ilha Solteira (SP). E-mail: maxfaria@agr.feis.unesp.br. $\left({ }^{*}\right)$ Autor correspondente.

$\left(^{6}\right)$ Departamento de Fitotecnia, Tecnologia de Alimentos e Sócio-Economia, UNESP-Câmpus de Ilha Solteira. 


\section{INTRODUÇÃO}

Em 2005, a produção brasileira de tomate alcançou aproximadamente 3,3 milhões de toneladas, em uma área cultivada de aproximadamente 58,4 mil hectares (FAOSTAT, 2006). O cultivo do tomateiro, caracterizado pelo uso intensivo de insumos e de mãode-obra, está inserido em um mercado dinâmico, com acentuadas variações estacionais de oferta e preço, obrigando o olericultor a um rigoroso planejamento da produção.

A utilização de abrigos para cultivo protegido pode representar uma excelente alternativa, na produção de frutos in natura, para o plantio de outonoinverno no Sul, Sudeste e Centro-Oeste, uma vez que, para culturas como o tomate, a demanda de mercado mantém-se estável nesses períodos (CAMARGo FilHO e MAzZeI, 2000). Todavia, o emprego de estruturas de proteção às plantas envolve custos adicionais, exigindo que essas áreas sejam intensivamente cultivadas e a relação custo-benefício seja otimizada pelo uso de técnicas adequadas.

Dentre essas técnicas, o manejo da densidade de plantas e da poda apical interferem na produtividade, na duração do período de colheita e na qualidade dos frutos de tomateiro de crescimento indeterminado. Segundo ANDRIOLo (1999), altas densidades de plantas, iguais ou superiores a cinco plantas por metro quadrado, podem se constituir em alternativa de manejo para concentrar a produção do tomateiro, quando associadas à eliminação das partes mais jovens da planta através da poda apical, sem prejuízo à produtividade, uma vez que não se alteram os índices de área foliar da cultura. Essas conclusões foram corroboradas pelos trabalhos de STRECK et al. (1998) e Hora (2003).

A produtividade do tomateiro, em ambiente protegido, está relacionada ao índice de área foliar da cultura, o qual interfere na interceptação da radiação solar pelo dossel (Papadopoulos e Pararajasingham, 1997). Neste aspecto, a utilização de cobertura plástica do solo pode se constituir em importante ferramenta, pois pode alterar o balanço de radiação para as plantas e para o solo, em função das características ópticas do material empregado (LiaKatas et al., 1986, DeCoteau et al., 1990, HAM et al., 1993, НAтT et al., 1994), com reflexos na produtividade e precocidade de colheita (ForTnUM et al., 1997, KASPERBAUER e Hunt, 1998).

Assim, o presente trabalho teve como objetivo estudar sistemas de cultivo para o tomateiro, resultantes de duas combinações de densidade de plantas e altura de poda apical, com número fixo de inflorescências por área, associados à utilização de coberturas plásticas do solo de diferentes cores, no que se refere a crescimento de plantas, produtividade, concentração de período de produção e qualidade de frutos de tomateiro, em ambiente protegido.

\section{MATERIAL E MÉTODOS}

O experimento foi desenvolvido de junho a novembro de 2003, na Fazenda de Ensino, Pesquisa e Extensão da Faculdade de Engenharia - UNESP, com latitude de $2022^{\prime} \mathrm{S}$, longitude de $51^{\circ} 22^{\prime} \mathrm{W}$ e altitude de $330 \mathrm{~m}$, no município de Ilha Solteira (SP), no interior de um abrigo para cultivo protegido com orientação leste-oeste, com dimensões de 5,4 x 30,0 m, pé-direito de $2,3 \mathrm{~m}$, teto em forma de arco, coberto com filme de polietileno transparente de $75 \mathrm{~mm}$ de espessura e fechamento lateral até $1,5 \mathrm{~m}$ de altura, com tela de sombreamento de $50 \%$.

O solo da área experimental foi classificado como Argissolo Vermelho, Eutrófico, conforme nomenclatura do Sistema Brasileiro de Classificação de Solos (Empresa Brasileira de Pesquisa Agropecuária - Embrapa/CNPSo, 1999). Os resultados da análise química do solo da área experimental indicaram $77 \mathrm{mg} \mathrm{dm}^{-3}$ de P resina; $18 \mathrm{~g} \mathrm{dm}^{-3}$ de M.O.; $\mathrm{pH}\left(\mathrm{CaCl}_{2}\right)$ de 6,$2 ; 1,1 \mathrm{mmol}_{\mathrm{c}} \mathrm{dm}^{-3}$ de K; $62 \mathrm{mmol}_{\mathrm{c}} \mathrm{dm}^{-}$ ${ }^{3} \mathrm{de} \mathrm{Ca} ; 16 \mathrm{mmol}_{\mathrm{C}} \mathrm{dm}^{-3} \mathrm{de} \mathrm{Mg}, 15 \mathrm{mmol}_{\mathrm{c}} \mathrm{dm}^{-3} \mathrm{de} \mathrm{H}+\mathrm{Al}$, SB de 79,1 $\mathrm{mmol}_{\mathrm{C}} \mathrm{dm}^{-3}$; CTC de $94,1 \mathrm{mmol}_{\mathrm{C}} \mathrm{dm}^{-3} \mathrm{e} \mathrm{V} \%$ de 84 .

Estudou-se o desempenho do híbrido Duradoro (EMBRAPA), de crescimento indeterminado e frutos longa-vida, sob dois sistemas de cultivo com haste única: SC1 - poda apical após a $5 .^{\mathrm{a}}$ inflorescência e 20.000 plantas ha ${ }^{-1}$ e SC2 - poda apical após a $2 .^{a}$ inflorescência e 50.000 plantas ha ${ }^{-1}$, e três condições de cobertura do solo: MB - filme de polietileno branco, com $50 \mathrm{~mm}$ de espessura, MP - filme de polietileno preto, com $50 \mathrm{~mm}$ de espessura e $\mathrm{SN}$ - solo nu, como testemunha. Para o estabelecimento do sistema de cultivo SC1, utilizou-se espaçamento de $0,5 \mathrm{~m}$ entre plantas e 1,0 m entre linhas; para o sistema de cultivo SC2, o espaçamento empregado foi de $0,2 \mathrm{~m}$ entre plantas e 1,0 m entre linhas.

Adotou-se o delineamento em blocos ao acaso, em esquema de análise de variância para parcelas subdivididas, com cinco repetições, 24 plantas por parcela (cobertura de solo) e 12 plantas por subparcela (sistema de cultivo). Os dados foram submetidos à análise de variância (Teste $\mathrm{F}$ ) e os efeitos dos tratamentos foram comparados pelo teste de Tukey a $5 \%$ de probabilidade.

A semeadura foi feita em 25/6/2003, em bandejas de poliestireno expandido de 128 células, utilizando-se substrato organomineral comercial. Os 
canteiros foram confeccionados com dimensões de 0,5 $\mathrm{m}$ de largura, $28,0 \mathrm{~m}$ de comprimento e $0,15 \mathrm{~m}$ de altura e a adubação de plantio consistiu na aplicação de $1,2 \mathrm{~g} \mathrm{~m}^{-2}$ de $\mathrm{N} ; 6,1 \mathrm{~g} \mathrm{~m}^{-2}$ de $\mathrm{P}_{2} \mathrm{O}_{5}$ e $6,2 \mathrm{~g} \mathrm{~m}^{-2}$ de $\mathrm{K}_{2} \mathrm{O}$, de acordo com recomendações de RAIj et al. (1997), tendo como fontes o superfosfato simples, cloreto de potássio e a fórmula 04-14-08.

Foi utilizado sistema de irrigação por gotejamento, com instalação de duas fitas gotejadoras por canteiro, aplicando-se, em seguida, a cobertura plástica do solo às parcelas pertinentes. Foram empregadas fitas gotejadoras com vazão nominal de $3,8 \mathrm{~L} \mathrm{~h}^{-1} \mathrm{~m}^{-1}$, a $70 \mathrm{kPa}$ de pressão de serviço, com emissores a cada $0,30 \mathrm{~m}$. A cultura foi irrigada durante todo o ciclo, inicialmente, em três turnos diários de 10 minutos, que passaram a quatro turnos de 10 minutos, no início da frutificação.

O transplante das mudas ocorreu em 24/7/ 2003. Para o tutoramento de plantas, foram utilizados fitilhos duplos fixados a fios de arame, em forma de espaldeira simples.

Foram realizadas desbrotas semanais, a partir de 12/8/2003. A poda apical após a 2. ${ }^{a}$ inflorescência, nas plantas sujeitas ao sistema de cultivo SC2, teve início em 7/9/2003. Nas plantas do sistema de cultivo SC1, a poda apical após a $5 .{ }^{\mathrm{a}}$ inflorescência ocorreu a partir de $25 / 9 / 2003$.

As fertirrigações foram realizadas em 11/9 e $3 / 10 / 2003$ e consistiram na aplicação de 1,0 g planta ${ }^{-1}$ de nitrato de cálcio, 1,0 g planta ${ }^{-1}$ de nitrato de potássio e $0,05 \mathrm{~g}_{\text {planta }}{ }^{-1}$ de ácido bórico. Durante o período experimental, foram feitas capinas manuais nas parcelas sem cobertura plástica do solo, bem como os tratamentos fitossanitários necessários para o controle de pragas e moléstias.

O início da colheita foi em 3/10/2003, aos 69 dias após o transplante, estendendo-se até $17 / 11$ / 2003, quando foram colhidos todos os frutos maduros e os que estavam no início de maturação.

No interior do abrigo para cultivo protegido, foi instalada uma estação climatológica automática, com sistema de aquisição de dados CR10X (Campbell Scientific), com piranômetro SP Lite (Sci-Tec Instruments), e sensor de temperatura e umidade relativa do ar HMP 45C (Vaisala), o que permitiu a obtenção da densidade de fluxo de radiação, temperatura e umidade relativa do ar no período do experimento.

Avaliaram-se as seguintes características: a) altura média de plantas, aos 15 e 45 dias após o transplante (DAT); b) massa média de matéria fresca dos frutos; c) número médio de frutos por área; d) produtividade; e) distribuição dos frutos por classe de tamanho em função do diâmetro transversal (Ø), em: pequenos $(50 \leq \varnothing<65 \mathrm{~mm})$, médios $(65 \leq \varnothing<80$ $\mathrm{mm})$, grandes $(80 \leq \varnothing<100 \mathrm{~mm})$ e gigantes $(\varnothing>100$ $\mathrm{mm})$; f) eficiência de conversão da radiação solar global incidente em massa de matéria fresca de frutos (CocKshull et al., 1992).

\section{RESULTADOS E DISCUSSÃO}

A densidade de fluxo de radiação global, no interior do abrigo para cultivo protegido variou entre 1,45 e $22,94 \mathrm{MJ} \mathrm{m}^{-2} \mathrm{dia}^{-1}$, com média de $13,60 \mathrm{MJ} \mathrm{m}^{-2}$ $\mathrm{dia}^{-1}$, o que correspondeu a uma transmissividade média de $75,4 \%$, no período de $25 / 7$ a $17 / 11 / 2003$. As temperaturas mínimas do ar, registradas no ambiente protegido, se situaram entre 8,0 e $29,0{ }^{\circ} \mathrm{C}$, com valor médio de $17,5^{\circ} \mathrm{C}$, enquanto as máximas variaram entre 17,1 e $40,5^{\circ} \mathrm{C}$, com valor médio de 32,5 ${ }^{\circ} \mathrm{C}$. A umidade relativa mínima do ar variou entre $11,1 \%$ e $81,0 \%$, com média de $30,9 \%$, ao passo que a umidade relativa máxima se situou entre $46,6 \%$ e $95,9 \%$, com valor médio de $82,1 \%$.

Para todas as características avaliadas, não se verificaram efeitos significativos para a interação entre os fatores sistema de cultivo e coberturas de solo, entretanto, constataram-se efeitos significativos para cada fator isoladamente.

Em relação à altura média de plantas, não foram detectadas diferenças significativas entre os sistemas de cultivo estudados, aos 15 DAT (Tabela 1). Todavia, o tratamento $\mathrm{SC} 2$, com plantas mais adensadas, promoveu maior altura média de plantas, aos 45 DAT (Tabela 1), como conseqüência da maior competição por luz, à medida que as plantas se desenvolveram, resultados semelhantes aos observados por SiLva JúnIOR et al. (1992) e Hora (2003).

A cobertura de solo não influenciou significativamente o crescimento inicial das plantas, como avaliado aos 15 DAT (Tabela 1). Porém, aos 45 DAT, as médias observadas nas parcelas com cobertura do solo com filme plástico preto (MP) foram significativamente maiores que as obtidas nas parcelas com solo nu (SN), sem diferir, entretanto, daquelas nas parcelas cobertas com filme plástico branco (MB), como indicado na tabela 1. Essas médias ocorreram, provavelmente, por alterações no balanço de radiação refletida para o dossel da cultura, principalmente, na razão entre a radiação na faixa do vermelho e do vermelho-distante (Decoteau et al., 1990). 
Tabela 1. Altura média de plantas aos 15 e 45 dias após o transplante (DAT), para tomate 'Duradoro', cultivado em ambiente protegido, sob diferentes sistemas de manejo e cobertura do solo. Ilha Solteira (SP), 2003

\begin{tabular}{lcc}
\hline \multirow{2}{*}{ Fator } & \multicolumn{2}{c}{ Altura de plantas } \\
\cline { 2 - 3 } & 15 DAT & 45 DAT \\
\cline { 2 - 3 } Sistema de Cultivo & & \\
SC1 & $24,72 \mathrm{a}$ & $84,69 \mathrm{~b}$ \\
SC2 & $24,38 \mathrm{a}$ & $98,13 \mathrm{a}$ \\
CV $(\%)$ & 13,21 & 5,28 \\
Cobertura de solo & & \\
MP & $26,35 \mathrm{a}$ & $95,00 \mathrm{a}$ \\
MB & $25,12 \mathrm{a}$ & $90,50 \mathrm{ab}$ \\
SN & $22,18 \mathrm{a}$ & $88,72 \mathrm{~b}$ \\
CV $(\%)$ & 19,67 & 5,34 \\
\hline
\end{tabular}

Médias seguidas pela mesma letra, nas colunas, dentro de cada fator, não diferem entre si pelo teste de Tukey $(\mathrm{P}>0,05)$.

SC1: Poda apical drástica após a $5 .^{\mathrm{a}}$ inflorescência e 20.000 plantas ha ${ }^{-1}$ e SC2: Poda apical drástica após a $2 .^{\text {a }}$ inflorescência e 50.000 plantas ha ${ }^{-1}$

MP: Cobertura do solo com filme plástico de polietileno, com 50 $\mathrm{mm}$ de espessura, na coloração preta; MB: Cobertura do solo com filme plástico de polietileno, com $50 \mathrm{~mm}$ de espessura, na coloração branca; $\mathrm{SN}$ : solo nu, como testemunha.

O tratamento com poda apical após a $2 .^{a}$ inflorescência e 50.000 plantas ha ${ }^{-1}$ (SC2), resultou em maior número de frutos por área $\left(60,1\right.$ frutos $\left.\mathrm{m}^{-2}\right) \mathrm{e}$ maior produtividade $\left(9,59 \mathrm{~kg} \mathrm{~m}^{-2}\right)$, sem que fossem detectadas, todavia, diferenças significativas na massa de matéria fresca média dos frutos (Tabela 2). Esses resultados divergiram daqueles constatados por HoRA (2003) que, avaliando os mesmos sistemas de cultivo estudados no presente ensaio, não observou diferenças significativas entre eles, no que se refere à produtividade e ao número de frutos por área, resultado esperado, uma vez que, para ambos os tratamentos, foram fixadas 10 inflorescências $\mathrm{m}^{-2}$.

A utilização de cobertura de solo, por sua vez, não influenciou significativamente a massa de matéria fresca média dos frutos, o número de frutos por área e a produtividade (Tabela 2). Esses resultados diferenciaram-se dos obtidos por CASTELlane et al. (1993a) e CASTELLANE et al. (1993b), que observaram incrementos de produção do tomateiro com uso de cobertura plástica do solo, na região de Jaboticabal (SP).

O menor número de frutos por área e a menor produtividade, no tratamento com poda apical após a 5. ${ }^{a}$ inflorescência e 20.000 plantas ha ${ }^{-1}$ (SC1), podem ser explicados pelo alto índice de abortamento de flores das últimas inflorescências, cujo período de emissão e desenvolvimento coincidiu com dias consecutivos (18/ 9 a 2/10/2003) de altas temperaturas do ar, com máximas variando entre 30,0 e $40,2{ }^{\circ} \mathrm{C}$ e valor médio de $36,0^{\circ} \mathrm{C}$. Essa condição é inadequada à fixação de flores e frutos, como discutido por MAKIsHIMA (s.d.), LOPES e STRIPARI (1998) e FONTES e SILVA (2005).

No que diz respeito à evolução percentual da produção, ao longo do período de colheita, verificouse que a adoção do tratamento SC2 determinou a concentração da produção nas primeiras sete semanas (Figura 1). Esse resultado foi semelhante àqueles de STRECK et al. (1998) e Hora (2003).

Tabela 2. Produtividade média, massa média de matéria fresca de fruto, número médio de frutos por área e eficiência na conversão de energia radiante em massa de matéria fresca de frutos por área, obtidos para tomate 'Duradoro', cultivado em ambiente protegido, sob diferentes sistemas de cultivo e cobertura do solo. Ilha Solteira (SP), 2003

\begin{tabular}{|c|c|c|c|c|}
\hline Fator & $\begin{array}{l}\text { Produtividade } \\
\text { média de fruto }\end{array}$ & $\begin{array}{c}\text { Massa média } \\
\text { de matéria fresca }\end{array}$ & $\begin{array}{c}\text { Número médio } \\
\text { de frutos }\end{array}$ & $\begin{array}{c}\text { Eficiência } \\
\text { de conversão }\end{array}$ \\
\hline & $\mathrm{kg} \mathrm{m}^{-2}$ & $\mathrm{~kg}$ & frutos $\mathrm{m}^{-2}$ & $\mathrm{~g} \mathrm{MJ}^{-1}$ \\
\hline \multicolumn{5}{|c|}{ Sistema de Cultivo } \\
\hline SC1 & $7,14 \mathrm{~b}$ & $0,168 \mathrm{a}$ & $42,43 \mathrm{~b}$ & $4,54 \mathrm{~b}$ \\
\hline SC2 & 9,59 a & $0,160 \mathrm{a}$ & 60,11 a & 7,52 a \\
\hline $\mathrm{CV}(\%)$ & 13,87 & 6,19 & 10,97 & 14,10 \\
\hline \multicolumn{5}{|c|}{ Cobertura de Solo } \\
\hline $\mathrm{MP}$ & $8,29 \mathrm{a}$ & 0,169 a & $48,94 \mathrm{a}$ & $5,96 \mathrm{a}$ \\
\hline $\mathrm{MB}$ & $8,82 \mathrm{a}$ & 0,163 a & 54,24 a & $6,36 \mathrm{a}$ \\
\hline SN & 7,99 a & 0,159 a & $50,64 \mathrm{a}$ & 5,77 a \\
\hline $\mathrm{CV}(\%)$ & 11,96 & 7,96 & 14,99 & 12,27 \\
\hline
\end{tabular}

Médias seguidas pela mesma letra, nas colunas, dentro de cada fator, não diferem entre si pelo teste de Tukey (P>0,05).

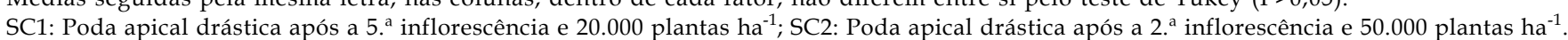
MP: Cobertura do solo com filme plástico de polietileno, com $50 \mathrm{~mm}$ de espessura, na coloração preta; MB: Cobertura do solo com filme plástico de polietileno, com $50 \mathrm{~mm}$ de espessura, na coloração branca; SN: solo nu, como testemunha. 


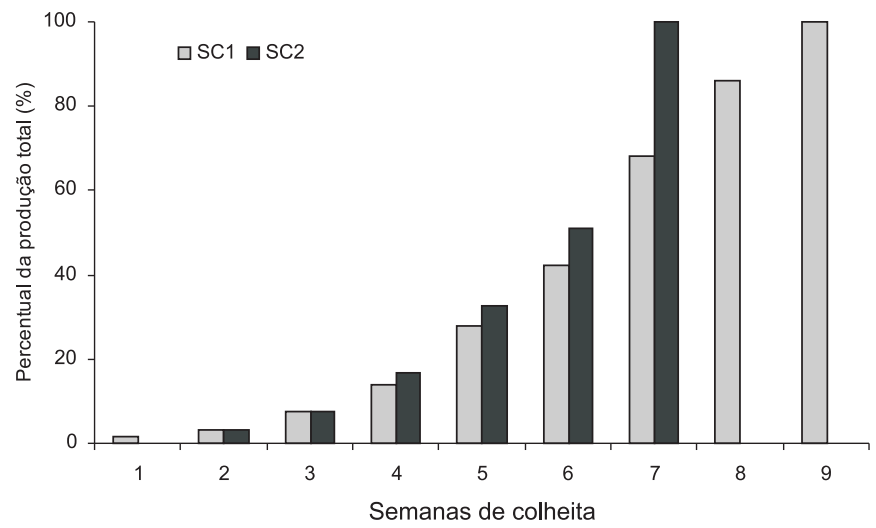

Figura 1. Evolução percentual da produção ao longo do período de colheita, obtida para os tratamentos SC1 (poda apical drástica após a 5. ${ }^{a}$ inflorescência e 20.000 plantas ha-1) e SC2 (poda apical drástica após a $2 .^{a}$ inflorescência e 50.000 plantas $\mathrm{ha}^{-1}$ ), para tomate 'Duradoro', em Ilha Solteira (SP), 2003.

Como reflexo das diferenças de produtividade e de período de colheita, entre os sistemas de cultivo estudados, observou-se que a utilização do tratamento SC2 permitiu maior eficiência na conversão de energia radiante em massa de matéria fresca de frutos, em comparação ao tratamento SC1, conforme a tabela 2. $\mathrm{O}$ valor de eficiência de conversão, de 7,52 $\mathrm{g} \mathrm{MJ}^{-1}$, ficou próximo dos valores máximos constatados por
STRECK et al. (1998), para as densidades de 80.000 e 100.000 plantas ha ${ }^{-1}$, com três inflorescências por planta, e, também, para a densidade de 40.000 plantas $\mathrm{ha}^{-1}$, com sete inflorescências por planta.

Não foi constatada influência significativa da cobertura do solo na eficiência de conversão da energia (Tabela 2). Esse resultado contrariou a expectativa de que a utilização de filmes plásticos refletivos (cor branca) pudesse alterar, favoravelmente, o balanço de energia para as plantas, com efeitos na produção.

O sistema de cultivo utilizado não interferiu na distribuição da produção, em virtude do tamanho dos frutos (Tabela 3), tendo sido constatado que, entre $75 \%$ e $80 \%$ da produção total, os frutos eram de tamanho médio, com diâmetro transversal entre 65 e $80 \mathrm{~mm}$, resultados discrepantes dos observados por CAmpos et al. (1987), que constataram que o aumento da população de plantas reduziu a produção de frutos com maior diâmetro.

A utilização de filme plástico preto, como cobertura do solo, diminuiu o percentual de produção de frutos pequenos (Tabela 3), sem que fossem verificadas diferenças significativas em relação aos demais tratamentos, nos valores obtidos para as demais classes de tamanho. Independentemente do tipo de cobertura do solo, a maior parte da produção foi obtida com frutos de tamanho médio.

Tabela 3. Dados transformados da distribuição percentual da produção, de acordo com a classificação dos frutos por tamanho, para tomate 'Duradoro', cultivado em ambiente protegido, sob diferentes sistemas de cultivo e coberturas do solo. Ilha Solteira (SP), 2003

\begin{tabular}{lcccc}
\hline & \multicolumn{2}{c}{ Dados transformados da distribuição percentual da produção de frutos $\left(^{1}\right)$} \\
\cline { 2 - 5 } Fator & $\begin{array}{c}\text { Pequenos } \\
50<\varnothing<65 \mathrm{~mm}\end{array}$ & $\begin{array}{c}\text { Médios } \\
65<\varnothing<80 \mathrm{~mm}\end{array}$ & $\begin{array}{c}\text { Grandes } \\
80<\varnothing<100 \mathrm{~mm}\end{array}$ & $\begin{array}{c}\text { Gigantes } \\
\varnothing>100 \mathrm{~mm}\end{array}$ \\
\hline Sistema de cultivo & 17,12 a $(8,08 \%)$ & 61,48 a $(75,64 \%)$ & 23,28 a $(15,48 \%)$ & $7,37 \mathrm{a}(0,80 \%)$ \\
SC1 & 16,59 a $(7,97 \%)$ & 64,75 a $(79,97 \%)$ & 20,02 a $(11,81 \%)$ & 6,19 a $(0,25 \%)$ \\
SC2 & 22,74 & 10,07 & 29,17 & 36,87 \\
CV $(\%)$ & & & & \\
Cobertura de solo & 13,89 b $(5,37 \%)$ & 64,11 a $(79,27 \%)$ & 22,85 a $(14,97 \%)$ & 6,45 a $(0,39 \%)$ \\
MP & 17,80 a $(8,91 \%)$ & 62,63 a $(76,79 \%)$ & 21,40 a $(13,95 \%)$ & 6,50 a $(0,35 \%)$ \\
MB & 18,88 a $(9,79 \%)$ & 62,60 a $(77,36 \%)$ & 20,69 a $(12,00 \%)$ & 7,39 a $(0,85 \%)$ \\
SN & 14,22 & 11,86 & 40,61 & 31,93 \\
CV $(\%)$ & & &
\end{tabular}

(1) Dados transformados em arc sen $\sqrt{\mathrm{x} / 100}$.

Médias seguidas pela mesma letra, nas colunas, dentro de cada fator, não diferem entre si pelo teste de Tukey (P>0,05).

Valores entre parênteses correspondem às médias originais, em percentagem, apenas para ilustração.

SC1: Poda apical após a 5. ${ }^{\mathrm{a}}$ inflorescência e 20.000 plantas ha-1; SC2: Poda apical após a 2. ${ }^{\text {a }}$ inflorescência e 50.000 plantas ha-1

MP: Cobertura do solo com filme plástico de polietileno, com $50 \mathrm{~mm}$ de espessura, na coloração preta; MB: Cobertura do solo com filme plástico de polietileno, com $50 \mathrm{~mm}$ de espessura, na coloração branca; SN: solo nu, como testemunha. 
Embora o material genético empregado no experimento seja caracterizado pela produção de frutos com 240 a $260 \mathrm{~g}$, foram obtidos frutos, em média, com massa de matéria fresca entre 160 e 170 $\mathrm{g}$ (Tabela 2), constituindo-se em amostra de que as condições de cultivo não permitiram a expressão do máximo potencial produtivo, em termos quantitativos e qualitativos.

$\mathrm{O}$ incremento no percentual de frutos de maior massa de matéria fresca, com diâmetro superior a $80 \mathrm{~mm}$, pode ter sido prejudicado pelas grandes amplitudes térmicas (até $20,8{ }^{\circ} \mathrm{C}$ ) observadas no período de florescimento e frutificação, acima daquelas indicadas como adequadas (entre 6 e $8^{\circ} \mathrm{C}$ ) por Filgueira (2000). Esse fato, bem como as pelas elevadas temperaturas máximas observadas no período, podem ter impedido a formação de frutos maiores, uma vez que, conforme MAKISHIMA (s.d.), temperaturas acima de $32{ }^{\circ} \mathrm{C}$ inibem o pegamento e o desenvolvimento do fruto. Esses resultados sugerem que tanto a densidade de plantas como a altura da poda apical (número de inflorescências) devem ser ajustadas às condições ambientais da época em que é realizado o cultivo.

\section{CONCLUSÕES}

1. A utilização de poda apical após a segunda inflorescência, associada à densidade de 50.000 plantas, possibilitou a concentração da colheita em um período de sete semanas e maior produtividade, devido ao menor abortamento de flores, em função da coincidência do período produtivo com época climaticamente mais adequada. Não houve efeito do sistema de cultivo sobre a massa média de matéria fresca e na distribuição dos frutos por classes de tamanho.

2. O emprego de cobertura plástica do solo não teve efeito sobre a massa de matéria fresca média de frutos, o número médio de frutos por área, a produtividade média e eficiência de conversão da radiação solar em massa de matéria fresca de frutos, porém, o uso de mulching com filme plástico preto reduziu a produção de frutos de tamanho pequeno.

\section{AGRADECIMENTOS}

À FAPESP, pela concessão de bolsa de iniciação científica, processo $\mathrm{n}^{\circ} 01 / 14228-6$, e à CAPES, pela concessão de bolsas de mestrado e doutorado

\section{REFERÊNCIAS}

ANDRIOLO, J.L. Fisiologia das culturas protegidas. Santa Maria: UFSM. 1999. 142p.

CAMARGO FILHO, W.P., MAZZEI, A.R. Abastecimento de legumes: tendência de preços. Informações Econômicas, São Paulo, v.30, n.10, p.35-49, 2000.

CAMPOS, J.P., BELFORD, C.C., GALVÃO, J.D., FONTES, P.C.R. Efeito da poda da haste e da população de plantas sobre a produção do tomateiro. Revista Ceres, Viçosa, v.34, n.192, p.198-208, 1987.

CASTELLANE, P.D.; ARAUJO, J.A.C. ; GABARRA, P.A.; HAYASHI, E.A.H. "Mulching" com filmes de polietileno de diferentes cores e controle de alguns insetos em tomateiro "Rio Grande" visando mercado "in natura". Horticultura Brasileira, Brasília, v.11, n.1, p.67, 1993a.

CASTELLANE, P.D.; ARAUJO, J.A.C. ; HAYASHI, E.A.H.; FERREIRA, M.E. Mulching com filme de polietileno negro e doses de N em cobertura de tomateiro 'Rio Grande' visando mercado 'in natura'. Horticultura Brasileira, Brasília, v.11, n.1, p.67, 1993 b.

COCKSHULL, K.E., GRAVES, C.J., CAVE, C.R.J. The influence of shading on yield of glasshouse tomatoes. Journal of Horticultural Science, London, v.67, n.1, p.11-24, 1992.

DECOTEAU, D.R., KASPERBAUER, M.J., HUNT, P.G. Bell pepper plant development over mulches of diverse colors. HortScience, Alexandria v. 25, n. 4, p. 460-2, 1990.

EMPRESA BRASILEIRA DE PESQUISA AGROPECUÁRIA Embrapa/CNPSo. Sistema Brasileiro de Classificação dos solos. Rio de Janeiro, 1999. 306p.

FAOSTAT Database Results. Disponível em: http:// www.fao.org/. Acesso em 10 de julho de 2006.

FILGUEIRA, F.A.R. Solanáceas II - Tomate: a hortaliça cosmopolita. In.:__. Novo manual de olericultura: agrotecnologia moderna na produção e comercialização de hortaliças. Viçosa: Universidade Federal de Viçosa, 2000. p.189-234.

FONTES, P.C.R.; SILVA, D.J.H. Cultura do tomate. In: FONTES, P.C.R. Olericultura teoria e prática. 1.ed. Viçosa: Departamento de Fitotecnia - Setor de Olericultura, 2005. p.457-475.

FORTNUM, B.A.; DECOTEAU, D.R.; KASPERBAUER, M.J. Colored mulches affect yield of fresh-market tomato infected with Meloidogyne incognita. Journal of Nematology, College Park, v.29, n.4, p.538-546, 1997.

HAM, J.M., KLUITENBERG, G.J., LAMONT, W.J. Optical properties of plastic mulches affects the field temperature regime. Journal of the American Society of Horticultural Science, Ashford, v. 118, n. 6, p. 188-93, 1993.

HATT, H.A. et al. Influence of spectral qualities and resulting soil temperatures of mulches films an bell pepper growth and production. Plasticulture, Paris, v 5, n.101, p. 13-22, 1994. 
HORA, R.C., Aplicação de luz na faixa do vermelho-extremo em mudas e diferentes sistemas de condução do tomateiro cultivado em ambiente protegido. 2003. 56p. Dissertação (Mestrado em Agronomia) - Faculdade de Engenharia UNESP, Ilha Solteira.

KASPERBAUER, M.J.; HUNT, P.G. Far-red light affects photosynthate allocation and yield of tomato over red mulch. Crop Science, Madison, v.38, n.4, p.970-974, 1998.

LIAKATAS, A. ; CLARK, J A.; MONTEITH, J.L. Measurements of the balance and soil heat flux, Agricultural and Forest Meteorology, Amsterdam, v.36, n.5, p.227-239, 1986.

LOPES, M.C.; STRIPARI, P.C. A cultura do tomateiro. In: GOTO, R.; TIVELLI, S.W. Produção de hortaliças em ambiente protegido: condições subtropicais. São Paulo: Fundação Editora UNESP, 1998. p.257-304.

MAKISHIMA, N. Efeitos dos fatores climáticos. Brasília: Centro Nacional de Produção de Hortaliças-CNPH, S.d. 5 p.
PAPADOPOULOS, A.P., PARARAJASINGHAM, S. The influence of plant spacing on light interception and use in greenhouse tomato (Lycopersicon esculentum Mill.): a review. Scientia Horticulturae, Amsterdam, v.69, p.1-29, 1997.

RAIJ, B.V.; CANTARELLA, H.; QUAGGIO, J.A.; FURLANI, A.M.C. Recomendação de adubação e calagem para o Estado de São Paulo. Campinas: IAC, 1997. 285p. (Boletim Técnico, 100)

SILVA JUNIOR, A.., MÜLLER, J.J.V., HONÓRIO, F.P. Poda e alta densidade de plantio na cultura do tomate. Agropecuária Catarinense, Florianópolis, v.5, n.1, p.57-61. 1992.

STRECK, N.A., BURIOL, G.A., ANDRIOLO, J.L., SANDRI, M.A. Influência da densidade de plantas e da poda apical drástica na produtividade do tomateiro em estufa de plástico. Pesquisa Agropecuária Brasileira, Brasília, v.33, n.7, p.1105-12, 1998. 